covered. A hall for demonstrations and lectures has just been erected, and we ventured to suggest that demonstrations of school-music would be welcome.

Special Institutions.-The work that is being done in schools for the blind, the deaf, and the mentally defective calls for respectful acknowledgment. Cases showing what is being accomplished, so far as material products are concerned, can be seen near the entrance. The moral benefit to the pupils cannot be expressed.

It reflects credit on the committee and the secretaries that the whole of this wonderful collection was in place at the opening of the exhibition. The objects are displayed in an admirable manner, and furnish innumerable suggestions of value to the practical teacher.

French Education Exhibit.-Aithough not large enough to furnish grounds for comparison of French with English organisation of education, the French section contains many interesting features. It is housed in the corridors between Shepherd's Bush and Wood Lane, unfortunately rather distant from the English section. The Écoles Professionelles, l'Enseignement Technique, and the Ecoles Primaires Supérieures contribute; Lille, Toulon, St. Etienne, Nîmes, Dupuy, and Rouen are represented.

Much of the manual work is excellent, and teachers of chemistry may glean some useful hints from the apparatus and diagrams, which are clearly displayed. There has been an attempt to introduce some really artistic adornment into certain of our own elementary schools; but we still have much to learn in this respect. Our authorities would do well to pay attention to the charming pictures sent by the Société Artistique de 1'Art à l'Ecole. Incidentally, we observed that Arabic was included in the curricula of some pupils whose note-books we inspected.

Undoubtedly the space allotted to the French education section is too small, and hence the display falls short of our expectations. We admit that those expectations were high. In justice to the work performed in bringing together the exhibit, we should add that the interesting quality of what we could see considerably strengthened our desire for a fuller display of recent achievements by our neighbours in the field of education.

G. F. Daniell.

\section{THE ELECTROCHEMISTRY OF LIGHT.}

$I^{N}$ the April and May numbers of the Journal of Physicab Chemistry, Mr. Wilder D. Bancroft contributes two long articles under this heading, long chiefly because of the very extensive quotations from the writings of Grotthuss, Herschel, H. W. Vogel, E. Vogel, Timiriazeff, Acworth, v. Huibl, Bothamley, and others whose work bears upon the subject. The object of the communication is " to bring the various catalytic actions of light under one head so far as possible," and to show that this may be done by accepting two laws enunciated by Grotthuss some ninety years ago:-(I) that only those rays of light which are absorbed can produce chemical action; (2) that the action of a ray of light is analogous to that of a voltaic cell. The action, therefore, is regarded as electrolytic, and sensitisers, whether " optical " or " chemical," are viewed as depolarisers. The fundamental conception of Grotthuss, that the action of light is essentially electrolytic in character, is held to be sound and to accord with modern notions, though the language in which he expressed it may be somewhat obscure.

The author proceeds to show that the decomposition of various salts containing silver, iron, copper, mercury, chromium, uranium, manganese, vanadium, and molybdenum, as the result of light action yields the same products as those resulting from electrolytic action, but that some substances are light-sensitive only in the presence of a suitable depolariser (or absorber of one of the products of the decomposition). Herschel's account of his experiments on the action of light upon iron salts and ferroand ferricyanides is quoted in full from the Philosophical Transactions. When paper is impregnated with a mixture of potassium ferricyanide and ferric chloride and exposed to light, the ferric chloride is reduced and Turnbull's blue is formed, further exposure giving a brown substance of unknown formula. The author records that since his writing Mr. Schluederberg has succeeded in producing this brown substance by electrolytic means. Herschel's observation that by the continued exposure of a Prussianblue print to light the colour was bleached, but that the colour returned when the print was left in the dark, and that this reversal took place even when the iron salt was exposed alone and the ferricyanide added afterwards, is explained by the supposition that the light, after it has reduced the iron of the ferric ammonium citrate to the ferrous state, by its prolonged action produces a reducing agent powerful enough to reduce the ferricyanide, the white ferrous ferrocyanide that results being re-oxidised in the dark.

The analogy between the oxidation of organic bodies by the action of light and by electrolysis is not so easy to trace for want of facts. Whether the oxygen (of the air) or the dye is the depolariser must be decided experimentally in each case, and "there is one conclusive way" of answering this question. "If the active light is light which is absorbed by the substance to be oxidised and not by the oxygen, then the substance to be oxidised has been made active by the light and the oxygen is the depolariser. If the active light is absorbed by oxygen and not by the substance to be oxidised, then this latter is the depolariser, and the oxygen is made active by light. If the active light is absorbed by both, it is possible that each is made active and that each is also the depolariser. In this last case, however, the results should be checked by experiments with another oxidising agent and another reducing agent. While light can only act in case it is absorbed, it does not follow that all light which is absorbed acts to any appreciable extent." In the bromination of organic compounds, Schramm and Zakrzewski have shown that the most effective rays correspond to the weaker bromine absorption bands in the yellow-green and orange instead of the stronger bands in the greenish-blue and blue. The researches of Herschel on the action of light on the colouring matter of flowers are explicable by the Grotthuss theory, and Timiriazeff in his Croonian lecture (1903) showed the strict applicability of the law so far as regards the correspondence between the absorption of light and its chemical action in the case of chlorophyll.

In some cases the depolariser changes the sensitiveness of the system with regard to certain rays. These substances are generally distinguished as "optical sensitisers," but, the author says, " a more rational distinction - would be between depolarisers with marked absorption bands and depolarisers without marked absorption bands." The discovery of the action of "optical sensitisers" by $\mathrm{H}$. W. Vogel in 1873 , that is, the possibility of sensitising photographic plates for the less refrangible rays by means of dyes, and Eder's work that led him to the conclusion that the absorption of silver bromide dyed with eosin and the maximum of the photographic sensitising action of eosin on silver bromide exactly coincide in the spectrum, are detailed by copious extracts from the writings of these investigators. Eder distinguished between the absorption of the dyed silver salt and a dyed gelatin film or aqueous solution of the dye, the former giving an absorption of greater wavelengths, in accordance with Kundt's law. Acworth, who worked under apparently ideal conditions, comparing the maxima of absorption and sensitiveness by estimating them in the same emulsion, found that the sensitiveness maximum was displaced towards the red as compared to the absorption maximum, and Wiedemann accounts for this by suggesting that the light at the place of maximum absorption may cause increased vibration within the molecule, resulting in radiations or heat waves, but without the amplitude of vibration in the molecule attaining a sufficient magnitude to result in any decomposition or chemical change of the molecule. Mr. Bancroft accepts Acworth's experimental results, but considers that his absorption curves show the sum of the absorptions of the dyed gelatin film and the dyed silver bromide, instead of the absorption - of the latter alone, and that therefore his results do not disprove Eder's conclusions that the maxima of absorption and photographic effect coincide.

Concerning the mode of action of such sensitisers, he states that the theory of Grotthuss enables us to make a definite statement with regard to them, "and one that differs to a certain extent from any of the previous ones.

NO. 2024, VOL. 78$]$ 
A sensitiser must be a depolariser, directly or indirectly. It nust be a reducing agent in the broad sense of the term, or it must be changed into one by the action of light. In either case the sensitiser is decomposed by the action of light on the sensitive plate." In support of this position he quotes Bothamley's observation that sensitisers act and a developable image is produced on exposure when the plate is immersed in a powerfully reducing solution, as proving that the dye is not oxidised by exposure as Abney suggested, and shows that the instability of the dye on exposure to light is generally acknowledged as a necessary condition for it to act as a sensitiser. This theory also explains Abney's experiment of exposing a collodion film stained with cyanine to the spectrum, and then coating it with a silver bromide emulsion and developing. The silver bromide was developed to an image over the absorption band of the cyanine, though the silver salt had never been exposed to light.

Among the general conclusions referring to this part of the subject that the author gives in his summing up, the two following have perhaps not been indicated in this abstract, namely:-(i) whether any substance is reduced or oxidised by light depends on the depolariser, and (2) al sensitisers are light sensitive, but the light sensitiveness and sensitising power need not run strictly parallel. Finally, he maintains that the electrochenical theory of light, first proposed by Grotthuss, accounts for all the known facts concerning the action of sensitisers and the action of light upon salts.

In a third chapter (Journal of Physical Chemistry, June) $\mathrm{Mr}$. Bancroft deals in a similar way with the action of chlorine and bromine on organic bodies, and the effect of halogen carriers upon the reactions in darkness and in light. $\mathrm{He}$ deals with benzene and toluene, and the formation of addition and substitution derivatives, distinguishing in the case of toluene between the replacement of hydrogen in the ring and in the side chain. After summing up the known facts and showing wherein other theories are deficient, he quotes the suggestion of Bruner that chain substitution is due to the bromine molecule and ring substitution to free bromine atoms, the preponderance of ring substitution when the reaction takes place in nitro benzene as a solvent being " certainly connected with the fact that bromine and a portion of the acid of the polybromides is dissociated into ions" in this solvent. Mr. Bancroft agrees with this theory in the main, but carries it further. He assumes that as light of suitable wavelength increases the conductivity of most gases, apparently by forming ions, this dissociation will probably give products (atoms or ions) one half of which will be positive and one half negative-whatever meaning may be attached to these words. If the dissociation products are ions, these words will have their usual meaning. To this assumption he adds four others that are partly new :- (2) That there is a slight reversible reaction between bromine and the positive gaseous ion,

$$
\mathrm{Br}_{2} \mathrm{Br}^{+} \leftrightarrows \mathrm{Br}_{2}+\mathrm{Br}^{+}
$$

(3) That substitutions take place in the ring when the negative gaseous ions are present in excess; otherwise in the side chain. (4) That the addition products of benzene are to be considered as analogous to the chain substitution products of toluene. (5) That there is a reversible dissociation of the halogen carriers with formation of socalled gaseous halogen ions, and whether these ions are positive or negative depends on the nature of the carrier.

Granting these assumptions, at low temperatures and in the dark, $\mathrm{Br}_{2} \cdot \mathrm{Br}^{+}$will be formed, and therefore an excess of negative bromine ions and ring substitution. With rising temperature the proportion of positive to negative bromine ions increases, and sunlight will increase the dissociation of bromine and of the $\mathrm{Br}_{2} \cdot \mathrm{Br}^{+}$, until the ratio of positive to negative ions approaches unity, and then side-chain substitution takes place. With reference to the fifth assumption, in chlorides of iron, antimony, molybdenum, and aluminium, there is no doubt that chlorine is the negative radical. In iodine monochloride, phosphorus pentachloride, and sulphur chloride, there is evidence of a tendency to form positive gaseous chlorine ions. The author remarks that "it would strengthen the argument if it were possible to show why negative chlorine sub- stitutes in the ring and positive chlorine in the side chain, but I do not see any explanation which can be carried through." A weak point in the argument is the behaviour of aluminium chloride, which Goldschmidt and. Larsen have found to behave exactly like stannic chloride as a carrier, although no lower chloride of aluminium is known. But, on the other hand, Turrentine has found evidence of its existence by the electrolysis of a chloride solution using an aluminium anode, though the aluminous salt has not been isolated.

C. J.

\section{BAROMETRIC GRADIENT AND WIND FORCE. ${ }^{2}$}

THE relation between the wind and the pressure distribution is a fundamental question of dynamical meteorology. In qualitative form it is expressed by Buys Ballot's well-known law that in the northern hemisphere an observer standing with his back to the wind has the region of lowest pressure on his left. The idea of a quantitative relation between gradient and wind has been made familiar to meteorologists by the classic researches of Guldberg and Mohn. It must, however, be admitted that a comparison of the wind velocity calculated from the pressure gradient by the method given by these authors with anemometer readings has not given entirely satisfactory results. The discrepancies are generally attributed to surface friction, but this quantity has not proved amenable to theoretical treatment, a difficulty which seems to have barred the way for further progress along these lines.

We should expect the effects of surface friction to decrease rapidly with altitude, so that the results of kite and balloon ascents should yield a more suitable material for a comparison of theory and observation than the observations at ground level do. In the report before us, Mr. Gold has used the data accumulated by the Prussian Aëronautical Observatory for such a comparison. From the distribution of pressure he has computed the gradient velocity over Berlin by the formula given by Guldberg and Mohn for each day of the year 1905, and has set beside it the observed direction and velocity of the wind at rooo metres and 2000 metres above sea-level. The agreement at yooo metres is surprisingly close. In the preface Dr. W. N. Shaw says of it:- "The general result of the investigation is, in my opinion, to confirm the suggestion that the adjustment of wind velocity to gradient is an automatic process which may be looked upon as a primary meteorological law, the results of which are more and more apparent as the conditions are more and more free from disturbing causes, mechanical or meteorological."

Cases of discrepancy between observed and calculated values may thus be regarded as exceptional, and their special investigation promises an interesting field for research. The observations of wind made at the surface may perhaps also be utilised in this manner if, as $\mathrm{Mr}$. Gold suggests, we prepare a series of coefficients, applicable to the individual stations, which will enable us to allow for the disturbing effects of surface friction.

For work on these lines a simple means of calculating gradient velocities from pressure distribution is required, and a considerable section of the report is accordingly devoted to providing it. Tables have been constructed showing the gradient velocities corresponding with different distances between consecutive isobars on charts of certain specified scales in different latitudes, or, if we prefer it, we have in the frontispiece a convenient scale for reading off the values from the map of the daily weather report or the working chart of the Meteorological Office.

The tables and scale give the gradient velocity on the assumption that the isobars are straight, i.e. that the path of the wind is a straight line. If this condition is not fulfilled, a simple correction has to be applied from a second set of tables (or scale of concentric circles) to allow for the curvature of the path of the wind.

There is one point of principle which requires to be mentioned in this connection. In applying the correction, I Report to the Director of the Meteorological Office on the Calculation of Wind Velocity from Pressure Distribution and on the Variation of the Meteorological Elemen's with Altitude. By Ernest Gold. (Lcndon Wyman and Sons, for H.M. Stationery Office. Price 2s. $6 i$. 